Volume 9, No.4, July - August 2020

International Journal of Advanced Trends in Computer Science and Engineering

Available Online at http://www.warse.org/IJATCSE/static/pdf/file/ijatcse06942020.pdf

https://doi.org/10.30534/ijatcse/2020/06942020

\title{
Enhanced Traffic Scheduling Algorithm by Implementing Committed Information Rate (CIR) in Broadband Systems for a Disaster-stricken Footprint in Telecommunications
}

\author{
Aaron Don M. Africa, Fernando M. Dimaala \\ Department of Electronics and Communications Engineering \\ De La Salle University, Manila \\ 2401 Taft Ave., Malate, Manila 1004, \\ Philippines, aaron.africa@dlsu.edu.ph
}

\begin{abstract}
In an emergency or disaster scenario, the need to communicate will be critical in saving a life; the telecommunication facilities are expected to deliver even though affected as well. Consequently, when signal traffic increases, service providers do not have enough capacity to support such as DRP only provides connectivity assurance. There is a need to establish an alternative and efficient broadband telecommunication network with consideration to channel estimation and has high transport capacity. The scheduling scheme plays an important role in the quality of service (QoS) provision.
\end{abstract}

IEEE 802.16 specification does not describe the scheduling scheme. The existing models involved tagging the packets with QoS parameters. This will always ensure that prioritization will take place based on this labeling scheme. In an emergency scenario, it will post a different challenge as a new "priority" type of information needs to be processed given its urgency. The network needs to schedule this traffic appropriately and strike a balance between processing the requirements dictated by its tagging and the given channel capacity, ensuring that the packets of information will have acceptable allocation. This paper proposes an algorithm model to support the latter requirements based on Committed Information Rate (CIR) experimentally performed in a testbed environment.

Key words: Committed Information Rate (CIR), Broadband, Telecommunication Systems, System Footprint.

\section{INTRODUCTION}

The telecommunication industry's footprint has been very dynamic and promising at an interesting rate. Manufacturers and vendors have entered the industry, each offering its technological uniqueness through state-of-the-art equipment and professional services. New technologies, such as Multi-Protocol Label Switching - Traffic Engineering (MPLS - TE), Internet Protocol (IP), Multiple Input Multiple Output (MIMO) antenna diversity and Dense Wavelength Division Multiplexing (DWDM) running on gigabit optical-fiber transmission systems have been developed and are being implemented at a rapid pace by major service providers $[1,2,3]$. Systems based on these emerging technologies are being put into place by carriers to serve the subscriber's demand for mobility, higher capacity, and reliability [4].

\section{WIMAX ARCHITECTURE}

The network is IP-based end-to-end and can be split into three major components: Mobile Stations (MS), also called Subscriber stations (SS) in the Fixed WiMAX specification; Access Service Network (ASN), which provides over-the-air connectivity, backhaul services, and WiMAX specific features such as encryption and authentication via the Public Key Management (PKM) module, handover support, radio resource management and Quality of Service (QoS); and the Connectivity Services Network (CSN), which is the point of connectivity to the Internet and corporate networks and provides AAA (Authorization, Authentication and Accounting) services, IP address assignment, QoS configuration and Mobile IP [5].

WiMAX has very versatile PHY and MAC layers. While the PHY layer has three distinct specifications (SC, OFDM and OFDMA), the MAC layer implements several advanced features such as encryption, security, error correction, link adaptation, power control, automatic retransmission, and quality of service [6].

The focus of this initiative is on the interface between MS and BS entities, also called the R1 reference point in WiMAX lingo, which is where the downlink and uplink scheduling takes place. When an MS connects to the network, a Policy Function residing on the CSN provides the ASN all the classification rules to assign MS' specific traffic to different QoS parameters. Based on such classification rules, the ASN gateway will set up service flows (one per classification rule, in each direction) to differentiate the traffic, and can even perform marking of the IP packets using DSCP (Differentiated Services Code Point) to reflect the different expected treatment of the packets over the backhaul. These 
service flows are reflected at the BS entity as logical connections (called CID, Connection Identifier) to each MS, each one having a specific scheduling service, according to the defined classification rules [7].

WiMAX brings about a novel concept to wireless systems: Per-flow quality-of-service. A single subscriber can have different streams of traffic, each one classified and scheduled over the air based on its quality-of-service parameters. Scheduling decisions get particularly complex as additional factors are considered:

1. Different supported modulation and coding schemes (MCS) as well as antenna techniques such as MIMO Matrix $\mathrm{A} / \mathrm{B}$.

2. RF conditions might suddenly change, which will change the MCS and hence the amount of bandwidth that can be allocated on each transmission opportunity.

3. Support for error correction methods such as Adaptive Repeat Request (ARQ) and Hybrid ARQ (H-ARQ).

4. PHY multiplexing scheme (OFDM vs. OFDMA)

In the case of WiMAX OFDM, the questions below must be answered, which can be as often as every two milliseconds. It is then crucial for the overall network performance to have a scheduling solution that not only optimizes the costly air link resources but is also efficient and runs within such demanding constraints.

1. What criteria should be used to decide the next packet to be scheduled?

2. What modulation shall be used for that specific packet?

Extensive research on scheduling solutions for other transmission technologies has been conducted in the past. Initial scheduler proposals for WiMAX OFDM are based, at least conceptually, on those solutions. The chosen scheduling algorithms were found to be the most relevant available in the literature for WiMAX OFDM, answering the two fundamental questions outlined above as well as presented considerations for supporting multiple QoS scheduling services [8]. WiMAX owes its QoS versatility to the different scheduling services supported, which determine how the network will allocate UL and DL transmission opportunities as well as how the MS can request uplink resources.

\section{OBJECTIVES OF THE STUDY 3.1. General Objective}

This paper aims to study the available scheduling in IEEE 802.16 systems and present an improved traffic scheduling algorithm to prevent bandwidth starvation by guaranteeing each connection's minimum bandwidth requirement and fairly allocate residual bandwidth to all connections.

\subsection{Specific Objectives}

The following, in particular, will be the purpose of the study:

1. To identify the scheduling algorithm design criteria

2. To provide analysis on existing traffic scheduling strategies
3. To elaborate on the strengths and weaknesses of the current capacity algorithm

4. To simulate the capability of existing algorithms

5. To validate the acceptability of the proposed algorithm

\subsection{Scope and Delimitations}

This research will involve assessing the existing channel allocation or traffic scheduling algorithm based on available standards. This will be complemented by simulation validation focusing on the description of scheduling and resource allocation algorithms. A new algorithm will be presented, and its starvation prevention performance will be evaluated using the existing QoS standard; specifically, for UGS, rtPS, nrtPS and BE class. These will be performed through a simulation tool and validated bymonitoring applications and software. This research will not be considering details beyond the MAC layer of IEEE 802.16d.

\section{SIMULATION PROCEDURES}

This research assessed available techniques proposed to deal with the challenges of efficient scheduling of air resources in the WiMAX context and developing the required algorithm to test at least three of these techniques in the downlink direction, namely Proportional Fairness (PF), Modified Largest Weighted Delay First (MLWDF) and Highest Urgency First (HUF), using available WiMAX simulator in the industry. The proposed algorithm analyzed using five key performance indicators (KPIs: average sector throughput, application throughput, average completion time, and fairness index) defined by the WiMAX Forum, an industry-led consortium promoting the WiMAX ecosystem [9].

A scheduler with a good average sector throughput should yield equivalent results in terms of application throughput, particularly for the FTP and HTTP Applications, in this research that can make use of extra bandwidth if available. The average completion time and fairness index, both based on the performance of the FTP application, are also interrelated. The MATLAB platform will be used to validate the situations and scenarios that will emulate the behavior of broadband subscribers with a different class of service.

\section{ALGORITHM DESIGN}

A simplified model of the scheduling end-to-end process with emphasis on the scheduling function provided at the Base Station (BS) entity. The network is IP-based end-to-end and can be split into three major components: Mobile Stations (MS), also called Subscriber stations (SS) in the Fixed WiMAX specification; Access Service Network (ASN), which provides over-the-air connectivity, backhaul services and specific features such as encryption and authentication via the Public Key Management (PKM) module, handover support, radio resource management and Quality of Service (QoS); and the Connectivity Services Network (CSN), which is the point of connectivity to the Internet and corporate networks and provides AAA (Authorization, Authentication 
and Accounting) services, IP address assignment, QoS configuration and Mobile IP.

WiMAX has very versatile PHY and MAC layers. While the PHY layer has three distinct specifications (SC, OFDM and OFDMA), the MAC layer implements several advanced features such as encryption, security, error correction, link adaptation, power control, automatic retransmission, and quality of service. The focus of the proposed algorithm is on the interface between MS and BS entities, also called the R1 reference point in WiMAX lingo, which is where the downlink and uplink scheduling takes place.

When an MS connects to the network, a Policy Function residing on the CSN provides the ASN all the classification rules to assign MS' specific traffic to different QoS parameters. Based on such classification rules, the ASN gateway will set up service flows (one per classification rule, in each direction) to differentiate the traffic, and can even perform marking of the IP packets using DSCP (Differentiated Services Code Point) to reflect the different expected treatment of the packets over the backhaul. These service flows are reflected at the BS entity as logical connections (called CID, Connection Identifier) to each MS, each one having a specific scheduling service, according to the defined classification rules. Figure 1 shows the end to end process scheduling.

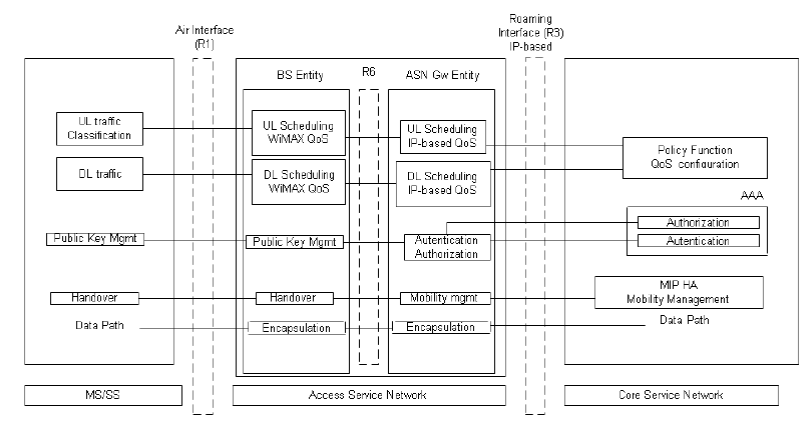

Figure 1: Scheduling End to End Process

Physical queues are maintained for the downlink direction for each scheduling service, while virtual queues are maintained in the uplink direction based on bandwidth requests coming from the mobiles or the configured sustained traffic rate for UGS and ertPS. It is up to the scheduler in each direction to sort through those queues and pick a subset of PDUs to be scheduled. Uplink packets update the virtual queues and uplink modulation and a feedback loop exists between the subscriber and the BS to identify the modulation to be used in the DL direction.

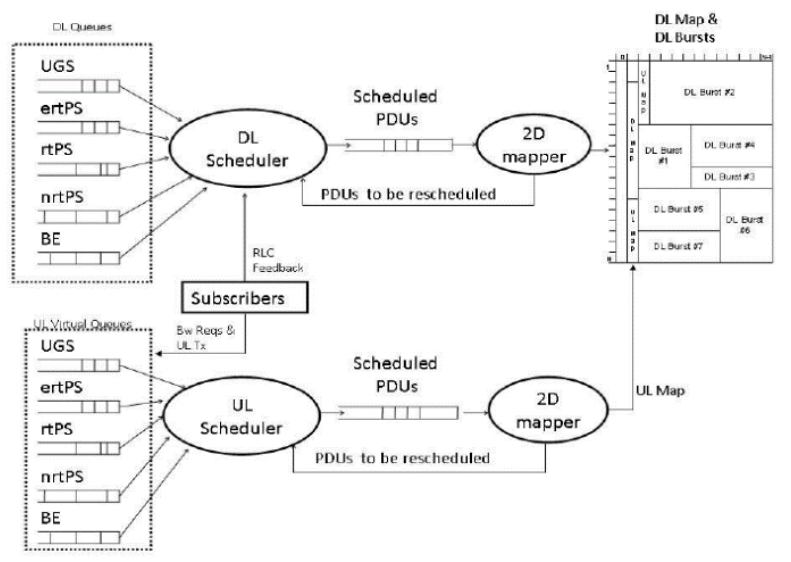

Figure 2: Framework Diagram

For the simulations in this research, the approach assumed the modulation as an attribute of the connection checked at scheduling time adopted. It is also important to highlight that while the process of 2D mapping is required for any implementation of an OFDMA scheduler; most papers do not elaborate on how their algorithm will build the DL/UL sub-frames and how to deal with unused slots and bumped PDUs due to frame fitting. Given such limitations, the current research will focus on the problem of choosing the PDUs to be scheduled over the air, conserving the frame fitting technique.

Table 1 shows the algorithm of the throughput

Table 1: Algorithm for throughput

\begin{tabular}{|c|c|l|}
\hline A & B & \multicolumn{1}{c|}{ Algorithm } \\
\hline 1 & 1 & $\begin{array}{l}\text { PF Algorithm used in CDMA EVD; it is } \\
\text { the prime example of this category as it } \\
\text { uses the feedback on the currently used } \\
\text { MCS, combined with the average data rate } \\
\text { used by each subscriber to determine } \\
\text { which packet gets access to the resources }\end{array}$ \\
\hline 1 & 0 & $\begin{array}{l}\text { Max SNR algorithm, which always serves } \\
\text { the terminal with the best RF conditions. } \\
\text { Although it optimizes overall throughput, } \\
\text { it doesn't have any fairness considerations }\end{array}$ \\
\hline 0 & 1 & $\begin{array}{l}\text { Round robin scheduling with time slots } \\
\text { assigned according to the average data rate } \\
\text { of each subscriber }\end{array}$ \\
\hline$n$ & 1 & $\begin{array}{l}\text { PF with data rate control. } n \text { is a } \\
\text { predetermined value that will control the } \\
\text { data rate allocated to subscribers. The } \\
\text { value of } \mathrm{n} \text { is the same for all subscribers } \\
\text { and hence different data rate limits cannot } \\
\text { be allocated }\end{array}$ \\
\hline $\mathrm{Ci}$ & 1 & $\begin{array}{l}\text { Adaptive PF. It complements the previous } \\
\text { algorithm by introducing Ci, a dynamic } \\
\text { data rate control value for user } i .\end{array}$ \\
\hline
\end{tabular}


Figure 3 shows the enforced minimum rate flowchart.

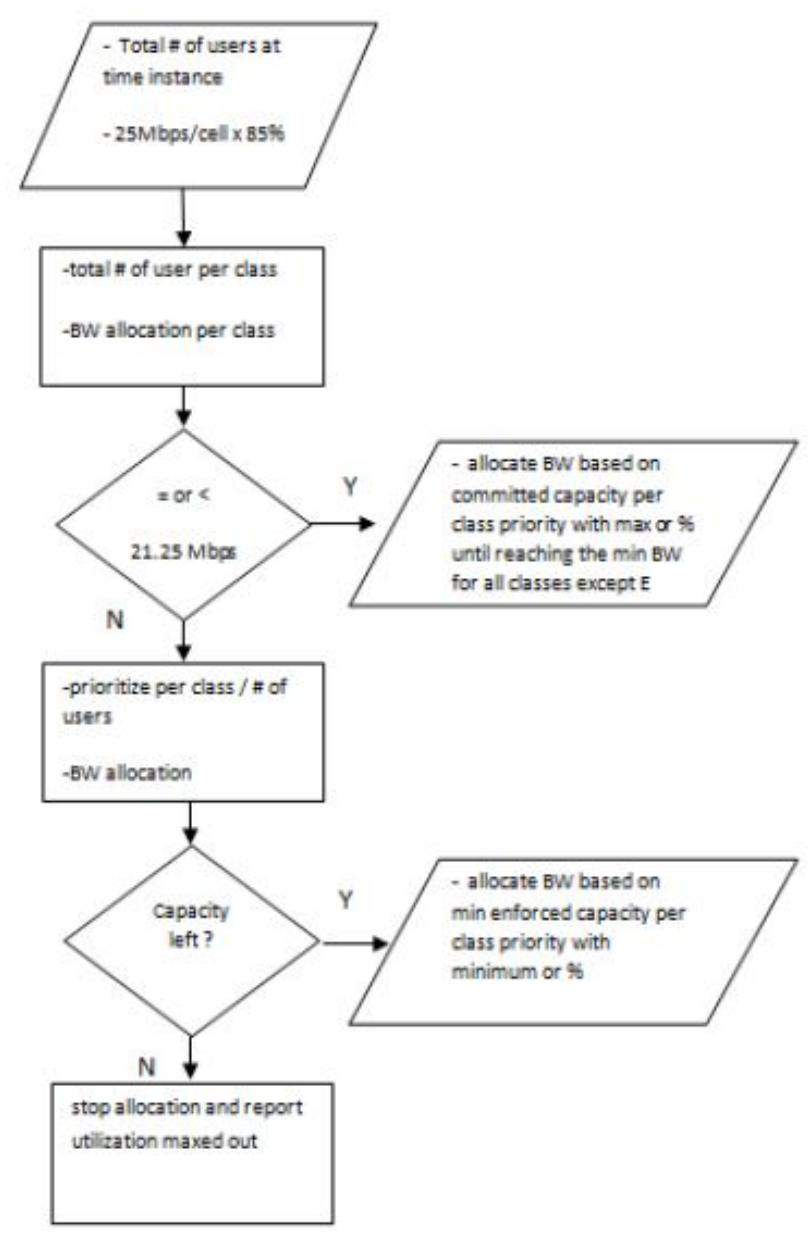

Figure 3: Enforced Minimum rate flowchart

\section{SIMULATION SURVEYS}

This thesis surveys recent techniques proposed to deal with the question of efficient scheduling of air resources in the WiMAX context and goes as far as developing the required algorithm to test at least three of these techniques in the downlink direction. The developed algorithms, together with Weighted Fair Queuing, analyzed using performance indicators defined by the WiMAX Forum, an industry-led consortium promoting the WiMAX ecosystem.

A scheduler with a good average sector throughput should yield equivalent results in terms of application throughput, particularly for HTTP, which is the only application simulated in this research that can make use of extra bandwidth if available.

\section{WiMAX SCHEDULER SIMULATION IN MATLAB}

The current industry practice is to allocate 25 Mbps of bandwidth to be shared by all users per WiMAX base transceiver station (BTS) or cell. The overlapping coverage implementation in the base station subsystem provides additional capacity and assurance that subscribers will be served and appropriately provided with voice and data resources. The available algorithm currently being used by the service providers supports all users, tagged to a certain subscription. There will be no further variant of subscribers available as compared with the WiMAX Forum recommendation of five classes (UGS, ertPS, rtPS, nrtPS and $\mathrm{BE})$. Generally, post-paid and pre-paid subscribers will be expected to make use of the mentioned capacity. Post-paid being any of the first four mentioned classes and pre-paid is the BE [10].

The existing types of subscribers under post-paid are limited to two options, usually rtPS (given a maximum of 1Mbps) and nrtPS (provisioned with a maximum of $0.512 \mathrm{Mbps}$ or 512Kpbs). The pre-paid subscribers are aligned to BE profile hence no commitment neither maximum nor minimum bandwidth allocation. The available algorithm will support the subscribers within the served area of a WiMAX base station transceiver strictly prioritizing the post-paid (rtPS and nrtPS respectively) users by allocating the majority of the bandwidth resource aligned to the subscribed profile not exceeding their maximum setting. Once that all the post-paid users have been supported, the remaining bandwidth resources will now be shared by all the $\mathrm{BE}$ or pre-paid users and will be in contention depending on the type of traffic being performed (browsing, file transfer, streaming, etc.) [11].

This will mean that the WiMAX access network will support and give priority to those classes of service with a certain level of commitment first and in the process creates bandwidth starvation for those classes with fewer bandwidth commitments and worst for those that do not have such assurance, also called best-effort class. The majority of subscribers presently supported in the Philippines are under the latter profile hence the main driver for this undertaking to possibly improve the scheduling mechanism of fairly and efficiently allocating the bandwidth resource [12].

The algorithm proposed for this study and will be simulated using the Mat LAB platform will be running on the following considerations.

Each WiMAX BTS will be provisioned with $25 \mathrm{Mbps}$, the available bandwidth though will only be $85 \%$ or $21.25 \mathrm{Mbps}$ to ensure equipment protection, such threshold is part of the HW protection specification [13].

There will be five classes of users to be present in the simulation running on the following maximum bandwidth capacity:

$$
\begin{aligned}
& \text { o Class A (UGS) at } 8 \mathrm{Mbps} \\
& \text { o Class B (ertPS) at } 5 \mathrm{Mbps} \\
& \text { o Class C (rtPS) at } 2 \mathrm{Mbps} \\
& \text { o Class D (nrtPS) at } 0.512 \mathrm{Mbps} \\
& \text { o Class E (BE) with no commitments }
\end{aligned}
$$

Enforced minimum rate reservation will be at $25 \%$ of the maximum bandwidth allocated for each class: 
o Class A (UGS) at 2Mbps

o Class B (ertPS) at 1.25Mbps

o Class C (rtPS) at $0.5 \mathrm{Mbps}$

o Class D (nrtPS) at $0.128 \mathrm{Mbps}$

o Class E (BE) with no commitments

1. An interval of $0.05 \mathrm{Mbps}$ will be used as granularity reference and limit will be at $0.000001 \mathrm{Mbps}$ to manage the resource allocation.

2. The presented algorithm source code in the earlier chapter will provide the tabular and graphical representation on how the new system thru the Mat LAB simulator set-up will allocate resources given different sets of quantities and types of users.

3. The simulator will show the results of the Optimized Bandwidth Allocation and will be compared with the output as well of the available scheduling methodology, describing and differentiating the manner of handling user demands, specifically as the algorithm enforces minimum bandwidth reservation and serve more subscribers.

4. - The simulated system will not allocate bandwidth if too much number of users demand resources even though it has enforced the minimum bandwidth reservation for all classes.

\section{DATA AND RESULTS}

The performance evaluation of scheduling algorithms must be through qualitative and quantitative metrics. This paper focused on the QoS important metrics namely throughput and fairness. The recommended classes of service were simulated emulating the possible scenarios if all types of classes of service that needs to be served by the same WiMAX base station transceiver.

To summarize, the following sets of scenarios reflecting the corresponding number of subscribers per class of service are the following:

a. Scenario 1 Class A - E $(1,2,1,1,5)$

b. Scenario 2 Class A - E $(1,1,3,1,37)$

c. Scenario 3 Class A - E $(1,1,2,5,18)$

d. Scenario 4 Class A - E $(3,4,5,6,7)$

e. Scenario 5 Class A - E $(4,6,8,10,15)$

f. Scenario 6 Class A - E $(4,6,8,11,35)$

g. Scenario 7 Class A - E (5, 6, 6, 6, 30)

h. Scenario 8 Class A - E $(6,10,2,5,30)$

i. Scenario 9 Class A - E (7, 10, 6, 6, 30)

The algorithm performs resource scheduling by identifying the current number of users given a certain time instance and from there provision the available bandwidth based on prioritization and fairness. The defined maximum bandwidth for a certain class of service will be provided with consideration to available resources the WiMAX base station transceiver may provide as planned. For scenarios 1 to 3 , the simulated system algorithm will limit the resource bandwidth available to $85 \%$ to ensure that hardware protection will be in place at that threshold as designed. The users for those classes with committed bandwidth allocation, namely UGS - Class
A, ertPS - Class B, rtPS - Class C and nrtPS - Class D, in these time instances were all assigned with their maximum values based on priority and fairness, what remains were evenly distributed to all the BE class of subscribers. These simulated results will be very much the same if subjected to the current industry situation or the existing algorithm. The same allocation will be given, and experience of the users will be within acceptable thresholds aligned to the type of premium they subscribed to. Matlab was used to simulate the system [14]. Figures 4 and 5 show the Matlab Simulation and the Pie Graph of the data.

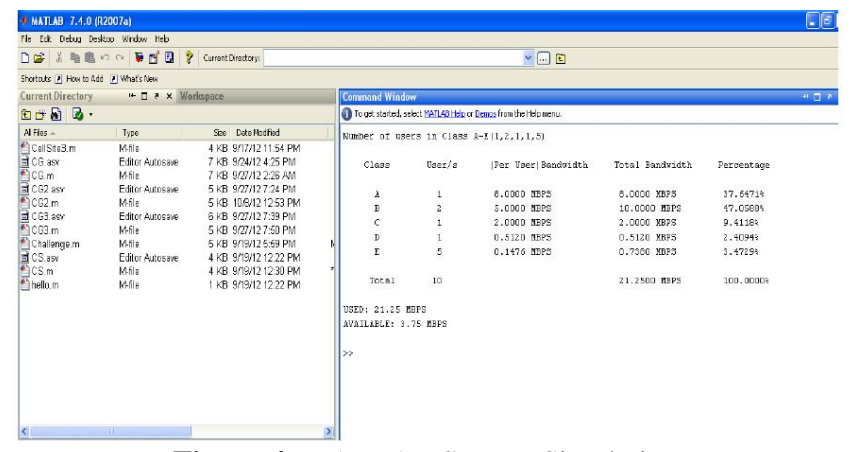

Figure 4: MATLAB System Simulation

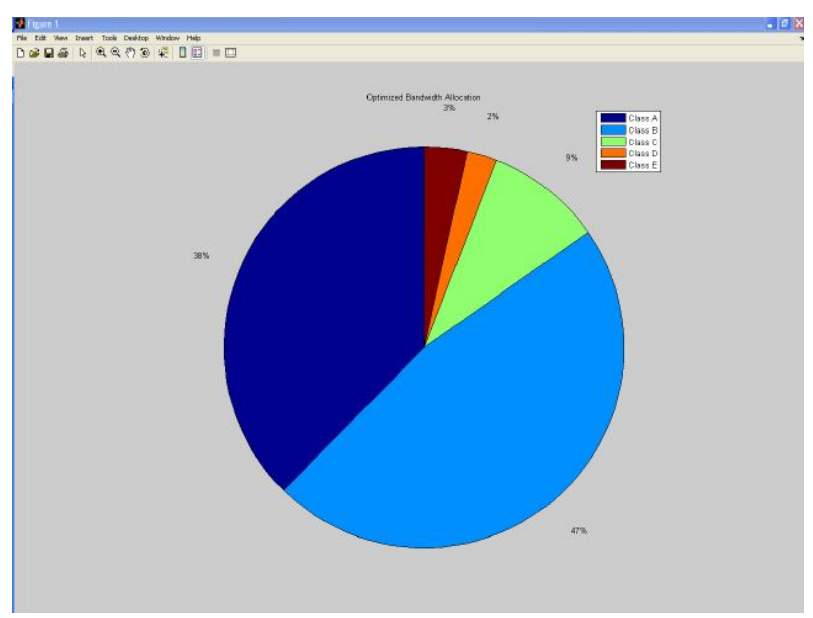

Figure 5: Pie Graph of the data

The scheduling algorithm will behave differently, and its value will be realized still to a certain extent as highlighted by the last three scenarios. The planned algorithm supports more users and provides acceptable bandwidth resources even to $\mathrm{BE}$ class of service especially in cases where there is less traffic or requirements from higher classes of requirements. This is where it effectively outperforms the status quo and ensures being novel in design. The output will be different if the proposed scheduling algorithm upon receiving all the requests especially from higher classes of service is more in numbers at a certain time instance. This was observed when we run the new algorithm using the given sets of subscribers under the scenarios with the following values $(5,6,6,6,30)$, $(6,10,2,5,30)$ and $(7,10,6,6,30)$ respectively.

The presented new scheduling algorithm will consistently enforce the designed $85 \%$ bandwidth availability to be 
allocated to all users as part of the hardware protection. The algorithm will then assess if the concern users at such time instance can be efficiently provisioned with acceptable bandwidth resources with consideration to established prioritization and fairness to lessen starvation to those class of service under a certain level of premium and those that are under best efforts profile. The proposed algorithm, upon enforcement of minimum bandwidth reservation for all the premium classes of service (belonging to UGS, ertPS, rtPS, and nrtPS) calculates that the available bandwidth will not be able to serve the requirements still will process the feasible scheduling methodology by selectively reserving minimum bandwidth for higher classes of service (specifically UGS and ertPS) but not anymore for rtPS and nrtPS. Scenario 7 reflects the mentioned situation and upon selecting to possibly display the outcome reveals the prioritization made and efficient allocation for the majority of given subscribers. The selective enforcement of minimum bandwidth reservation will be further noticed in the scenarios where worse conditions of having more high-end class of service or users will need to be supported requiring the least committed bandwidth and the remaining resources will be shared to the rest of users which effectively limit bandwidth starvation.

\section{CONCLUSIONS AND RECOMMENDATIONS}

The whole research effort revolved in understanding the WiMAX network especially on how this can be maximized in serving the broadband requirement of users with flexible communication needs. The technology is not mature though in serving a variety of subscriber's bandwidth and scalability requirements. Initial simulation activity for different service classes using the MAT LAB simulation tool reflects the natural behavior of known scheduling algorithms basing it on key performance metrics namely throughput and fairness. It can be observed that each of the four options has its unique applicability depending on the scenario. It can be concluded that these algorithms cannot serve multiple users to a point that no priority and capacity assurance.

The next simulation scenario observes the proposed scheduling concept in a multiple service class case and traffic characteristics. The activity was performed and simulated through MATLAB scheduling tool. The output reflects the capability of the base station transceiver to consider and prioritize the service class through the implemented scheduling algorithm anchored on the profile created at the ASN. The defined maximum and minimum bandwidth were achieved through a significant number of users were not explored due to the limitation of the test condition.

The last simulated activities reflected the capability of the algorithm still to prioritize traffic based on the hierarchy of users recommended by WiMAX Forum. Moreover, the difference of this scheduler to the other available algorithm is that it does not strictly serve all the priority traffic first and then distributes the remainder of the bandwidth to the rest of the low priority users which is where resource starvation comes in. It will continue to serve the priority service class to a committed minimum rate that is acceptable under that service class profile. It will not be the perfect algorithm though as can be seen in the last procedures because of the cell planning consideration, there will be a point that all the bandwidth resource will be used up, thru this algorithm through the output reflected its capability to serve more users, that is practicing fairness.

The proposed algorithm can support more users to a level that the optimized or planned cell capacity can offer. If there will be high priority users occupying the same sector or cell, the algorithm will need to decide to balance on service class priority and minimum requirements. In the future, it will be critical to also consider other quality performance metrics and align it to the 4G and higher broadband technology direction by the ITU.

\section{REFERENCES}

[1] R. Alroobaea, "An empirical combination of machine learning models to enhance author profiling performance," International Journal of Advanced Trends in Computer Science and Engineering. Vol. 9, No. 2, pp.2130-2137, 2020. https://doi.org/10.30534/ijatcse/2020/187922020

[2] G. Sowmya, N. Sirisha, N and G. D. Jyothi, "Use of big data to measure attentiveness of the student," International Journal of Advanced Trends in Computer Science and Engineering. Vol. 9, No. 2, pp. 2350-2352, 2020. https://doi.org/10.30534/ijatcse/2020/219922020

[3] T. Oo and A. Africa, "Design and implementation of bandwidth monitoring, line aggregation of VoIP," International Journal of Advanced Trends in Computer Science and Engineering. Vol. 8, No. 4, pp.1326-1331, 2019. https://doi.org/10.30534/ijatcse/2019/46842019

[4] A. Kailuke, P. Agrawal and R.V.Kshirsagar, "Design of Low Power, Low Jitter PLL for WiMAX Application in $0.18 \mu \mathrm{m}$ CMOS Process," Procedia Computer Science. Vol. 152, pp. 390-397, 2019.

[5] R. Sahoo and D. Vakula,"Gain enhancement of conformal wideband antenna with parasitic elements and low index metamaterial for WiMAX application," AEU International Journal of Electronics and Communications. Vol. 105, pp. 24-35, 2019.

[6] K. K. Naik, "Asymmetric CPW-fed SRR patch antenna for WLAN/WiMAX applications," AEU - International Journal of Electronics and Communications. Vol. 93, pp. 103-108, 2018. https://doi.org/10.1016/j.aeue.2018.06.008

[7] G.Vijayalakshmy and G.Sivaradje, "WiMAX-UMTS Converging Architecture with IMS Signalling Analysis to Achieve QoS," Procedia Technology. Vol. 6, pp.16-23, 2012.

[8] K.Nidhi Lal and A. Kumar, "ICN-WiMAX: An Application of Network coding-based Centrality-measures caching over IEEE 802.16," Procedia Computer Science.Vol. 125, pp. 241-247, 2018.

[9] C. M. K. Swain and S. Das, "Effects of threshold-based relay selection algorithms on the performance of an IEEE 
802.16j mobile multi-hop relay (MMR) WiMAX network," Digital Communications and Networks. Vol. 4, No. 1,pp. 58-68, 2018.

https://doi.org/10.1016/j.dcan.2017.09.003

[10] L. Kansal, V. Sharma and J. Singh, "Performance evaluation of FFT-WiMAX against WHT-WiMAX over Rayleigh fading channel," Optik. Vol. 127, No. 10, pp. 4514-4519, 2016.

[11] M.M.Eissa, "New protection principle for smart grid with renewable energy sources integration using WiMAX centralized scheduling technology," International Journal of Electrical Power \& Energy Systems. Vol. 97, pp. 372-384, 2018. https://doi.org/10.1016/j.ijepes.2017.11.014

[12] N.C.Wang, Y.K.Chiang and S.M.Wei,"RSVP Extensions for Seamless Handoff in Heterogeneous WLAN/WiMAX Networks," Journal of Applied Research and Technology. Vol. 11, No. 4,pp. 540-548, 2013.

[13] A. K. Gangwar and M. S. Alam, "A miniaturized quad-band antenna with slotted patch for WiMAX/WLAN/GSM applications," AEU International Journal of Electronics and Communications. Vol. 112,2019. https://doi.org/10.1016/j.aeue.2019.152911

[14] Matlab. https://www.mathworks.com/products/matlab.html. 2020. 